\section{SOBRE FULLERENOS, NANOTUBOS DE CARBONO Y GRAFENOS}

\author{
Nazario Martín \\ Departamento de Química Orgánica. Facultad de Ciencias Químicas. \\ Universidad Complutense de Madrid. E-28040 Madrid, Spain \\ nazmar@quim.ucm.es; http:/www.ucm.es/info/fullerene \\ IMDEA-Nanociencia. Facultad de Ciencias, Módulo C-IX, 3. ${ }^{a}$ planta. \\ Universidad Autónoma de Madrid. Ciudad Universitaria de \\ Cantoblanco, E-28049 Madrid, Spain
}

\section{INTRODUCCIÓN}

El año 2011 se celebra como el año internacional de la Química pero, curiosamente, en este año también se celebra el 70 cumpleaños de un gran científico, Fred Wudl, con quien tuve la suerte de comenzar mi andadura en la química de fullerenos en la costa californiana de la Universidad de Santa Barbara, durante el verano de 1994. Hoy, casi diecisiete años después, las nuevas formas en que se manifiesta el carbono me fascinan tanto o más que entonces.

El carbono es el único elemento de la Tabla Periódica capaz de reunir las propiedades necesarias para la aparición de la vida sobre la Tierra. Su capacidad de hibridación de orbitales atómicos, generando carbonos de naturaleza $s p^{3}-, s p^{2}-y s p-$, le confiere una situación única para la construcción de una amplia variedad de formas alotrópicas (1). Sin embargo, resulta sorprendente el hecho de que hasta hace tan solo 25 años, solo dos alótropos de carbono, el diamante -constituido por carbonos $s p^{3}-$ y el grafito -constituido por carbonos $s p^{2}$ - fuesen conocidos por la comunidad científica. Ambas formas alotrópicas de carbono presentan una estructura reticular con los átomos de carbono extendiéndose infinitamente en las tres direcciones del espacio.

Este escenario cambió drásticamente con el descubrimiento de los fullerenos (2) -jaulas cerradas constituidas exclusi-

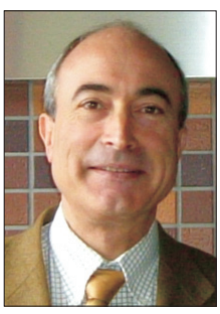

Este trabajo está dedicado a la memoria de Lucas Hernández.

vamente por un número definido de átomos de carbono con elevada simetría- seguido, en orden cronológico, por los nanotubos de carbono (3) -estructuras constituidas por átomos de carbono con forma cilíndrica- $y$, más recientemente, por los grafenos (4) -láminas bidimensionales de átomos de carbono-. Actualmente se conocen un número significativamente superior de "nanoformas" de carbono, tales como "fullerenos endoédricos", "nanocuernos de carbono", "nanocebollas de carbono", "nanotorus" (nanotubos de carbono formando un anillo), "nanovasos" (a modo de una serie de vasos apilados) o incluso los "nanocapullos" (formados por un fullereno que emerge de un nanotubo a modo de un capullo sobre un tallo) o los denominados guisantes (formados por un nanotubo de carbono conteniendo moléculas de fullerenos), configurando así un auténtico zoo de nuevas formas de carbono. A este grupo hay que añadir la forma más reciente de carbono conocida como grafenos (una lámina monoatómica de grafito) considerados materiales excepcionales que actualmente están recibiendo la atención de la comunidad científica internacional.

Este hecho en sí mismo, es decir, las numerosas formas en que el elemento carbono puede manifestarse en la actualidad, genera un primer problema taxonómico. Es preciso clasificar las estructuras anteriores como nuevos alótropos diferentes de Fullerenos o, por el contrario, deben de considerarse diferentes manifestaciones de los alótropos conocidos, de forma genérica, como fullerenos. 
La IUPAC en su Compendio de terminología química (2. ${ }^{\text {a edi- }}$ ción, 1997) define el concepto de alótropo como "las diferentes modificaciones estructurales de un elemento". Aunque esta definición de la IUPAC parece llevar a considerar las nuevas estructuras de carbono como nuevos alótropos, la comunidad científica ha tendido a considerar los fullerenos como la tercera forma alotrópica del carbono, en donde los átomos se unen entre sí dando lugar a formaciones esféricas, tubulares o elipsoidales, englobando así a todas estas formaciones en un mismo alótropo, los fullerenos. Para distinguir estas formaciones, en un artículo de nuestro grupo de investigación las denominamos "nanoformas de carbono" (5).
Sin embargo, el objetivo del presente trabajo no es una presentación general de las diferentes formas de los fullerenos, algunas de las cuales no pasan de ser actualmente más que una curiosidad científica. Por el contrario, este trabajo pretende centrarse en aquellas estructuras de carbono que más expectación han provocado en la comunidad científica por sus propiedades excepcionales así como por su belleza y simetría, cualidades estas últimas que han cautivado la imaginación de los químicos desde sus orígenes. En concreto, me refiero a fullerenos, nanotubos de carbono y grafenos (Figura 1).

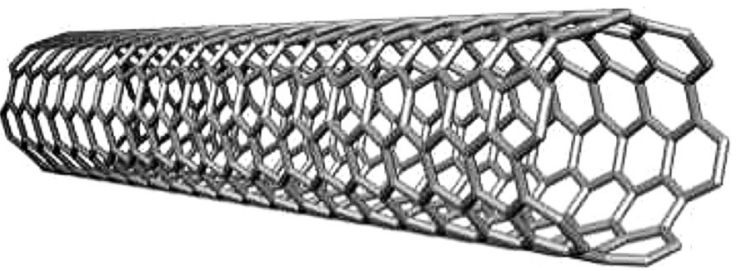

Figura 1. Estructura química del fullereno $C_{60}$, un nanotubo de carbono de pared sencilla y del grafeno

No obstante, y antes de pasar a hacer una presentación formal de estas nanoformas de carbono, dos de las cuales han merecido la concesión de sendos Premios Nobel a sus descubridores (Fullerenos, Premio Nobel de Química 1996 y Grafenos Premio Nobel de Física 2010) me gustaría hacer una reflexión sobre sus descubrimientos respectivos, como ejemplo de cómo los científicos vamos configurando la historia de nuestra ciencia. Frente a los científicos que se llevan la "gloria" debida por sus descubrimientos que, sin duda, hacen avanzar de forma real la ciencia, se encuentran otros científicos que han contribuido de forma significativa a ese avance científico pero cuyo reconocimiento queda en un segundo plano y, con el devenir de los tiempos, incluso olvidado.

Considérese, por tanto, lo que sigue, como un homenaje a todos aquellos científicos que fueron levantando las bases para que otros llegasen a ver más lejos. 


\section{Descubrimiento de fullerenos, nanotubos DE CARBONO Y GRAFENOS}

\subsection{Fullerenos}

Los fullerenos fueron descubiertos por Robert F. Curl, Sir Harold W. Kroto y Richard E. Smalley en 1985 (2). Tan solo once años después de este descubrimiento, los tres científicos recibieron el Premio Nobel de Química del año 1996 por "el descubrimiento de los fullerenos" (6). Previamente, en 1991, el fullereno fue declarado la molécula del año por la revista Science. Este descubrimiento ha dado lugar a un nuevo campo de estudio en diferentes ámbitos de la ciencia, creando una excitación en la comunidad científica pocas veces observada. Esta excitación ha rebasado el ámbito puramente científico y, por su descubrimiento inicial en Texas, en mayo de 1997 su asamblea declaró oficialmente al llamado buckminsterfullereno $\mathrm{C}_{60}$, la molécula estatal de Texas.

Las expectativas tanto en investigación básica como sus previsibles aplicaciones tecnológicas han hecho de los fullerenos, uno de los sistemas más estudiados en ciencia a nivel internacional. Por tanto, sus descubridores han pasado ya a la historia de la ciencia por derecho propio (Figura 2).
Sin embargo, los fullerenos pasaron a ser realmente compuestos químicos manejables y químicamente modificables a partir del método puesto a punto por el físico nuclear Wolfgang Krätschmer quien, en 1990, inventó un método para producir fullerenos a escala multigramo mediante la disolución del hollin obtenido en sus experimentos en benceno. Este hecho abrió, así, el estudio de estos compuestos a la comunidad química con su famosa publicación en la revista Nature titulado: "Solid $\mathrm{C}_{60}$ : A new form of carbón" (7).

Aunque el pasado 28 de abril Krätschmer recibió el European Inventor Award 2010 en la categoría de "lifetime achievement" por el desarrollo de la síntesis de fullerenos (European Patent Document EP 0500914B1 (1991/98)) de las manos de nuestro Príncipe D. Felipe de Borbón, esto no compensa lo que, a juicio de muchos, debió de ser merecedor del máximo galardón en ciencia. En palabras de Smalley "Had there not been a method to make it in measurable amounts, it would not have had an impact". También Curl tuvo unas palabras en reconocimiento del trabajo de Krätschmer y Huffman: "Huffman's work took it from mass spectrometers to the laboratory. It must have been a close decision by the Nobel committee over who should get it."
Figure 2. El equipo de Rice/Sussex el día antes de enviar su trabajo a Nature. (por la izquierda): O'Brien, Smalley, Curl, Kroto, and Heath.

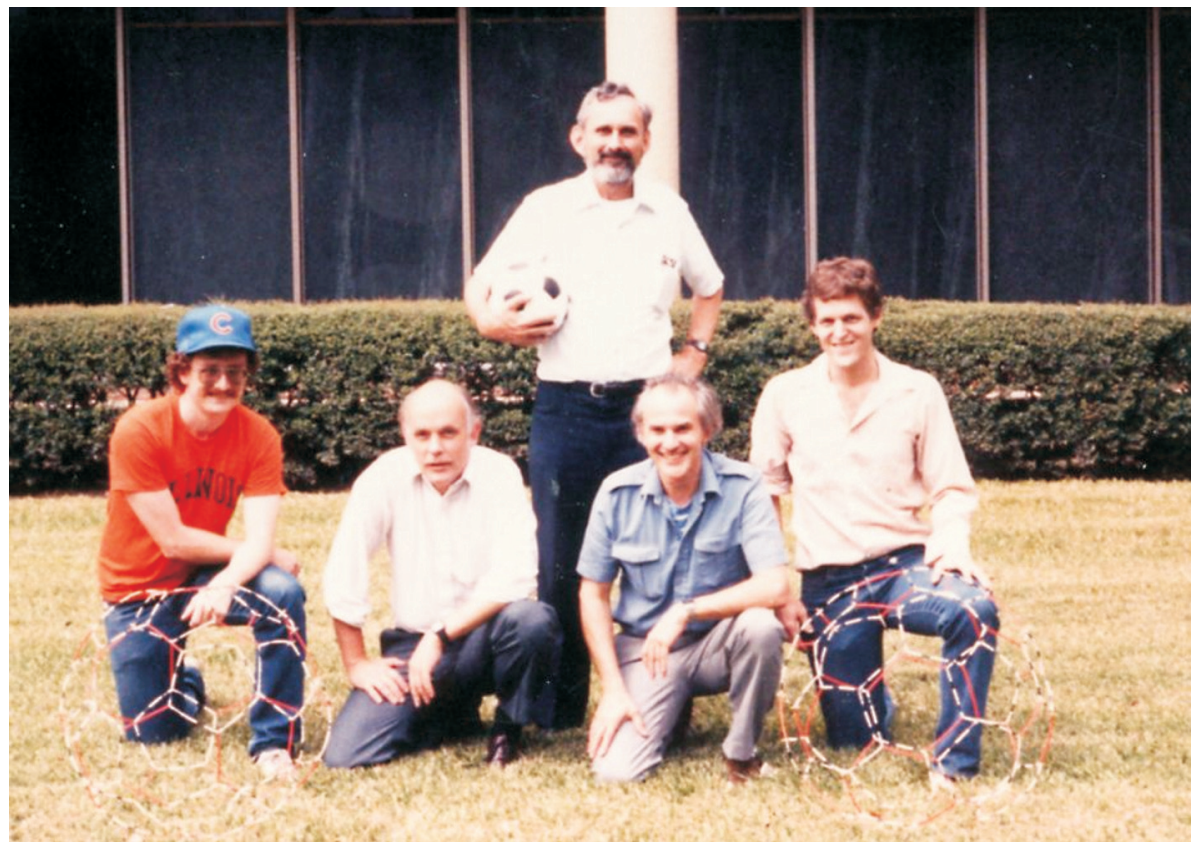

ARBOR CLXXXVII EXTRA 2011 115-131 ISSN: 0210-1963

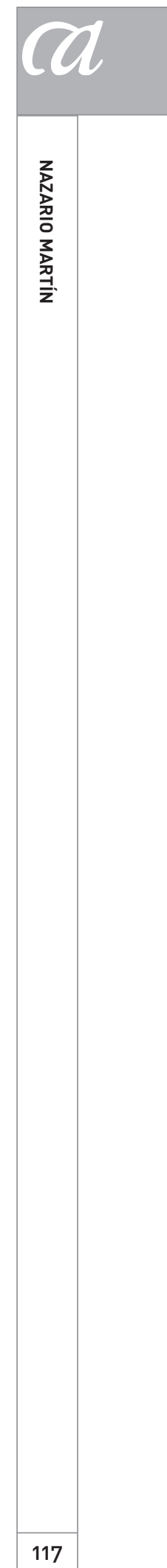


Sin embargo, la historia de los fullerenos se remonta mucho más atrás. De hecho, la molécula de $\mathrm{C}_{60}$ fue propuesta 15 años antes de su descubrimiento por Eiji Osawa, un químico japonés de la universidad de Kyoto, en un trabajo publicado en japonés (8). Kroto, quien no conocía el trabajo de Osawa en el momento de su descubrimiento, en parte por la barrera idiomática, ha dado, desde entonces, un gran crédito a este investigador.

También en 1970 R. W. Henson, entonces en el Atomic Energy Research Establishment, estudiando los daños de la radiación en los moderadores de grafito de los reactores nucleares, observó algunos modos de difracción de rayos- $X$ inusuales en fibras de carbono, y propuso la estructura e hizo un modelo de papel de la molécula de $\mathrm{C}_{60}$. La evidencia para esta nueva forma de carbono fue muy débil y no fue aceptada incluso por sus propios colegas. Los resultados nunca fueron publicados, aunque el modelo en papel aún se conserva y tuvo algún crédito posterior en la revista Carbon en 1999 (9).

La idea de una estructura en forma de jaula de carbono fue inicialmente propuesta por David E. H. Jones, en un artículo que escribió en el New Scientist con el nombre de "Daedalus" (New Sci. 1966, 32, 245). El sugirió que sería posible crear moléculas gigantes huecas distorsionando una red plana de carbonos hexagonales por adición de átomos de impureza. Esto, podria introducir la curvatura necesaria para llegar a una capa esférica. Sin embargo, él nunca llegó a explicar cómo se podría conseguir.

Algunos químicos algo más románticos han visto en Leonardo da Vinci el primer científico que "modelizó" la molécula de fullereno en su ya famosa ilustración para el libro de Luca Pacioli titulado "De Divina Proportione", publicado en Venecia en 1509. En esta ilustración puede verse el icosaedro truncado con forma de balón de futbol que Leonardo Ilamó Ycocedron Abscisus Vacuus (Figura 3).

\subsection{Nanotubos de carbono}

El descubrimiento de los nanotubos de carbono es quizás algo más complejo por la cantidad de artículos previamente publicados a aquellos que se consideran habitualmente los trabajos seminales que suscitaron el interés de la comunidad científica.
Tradicionalmente se asigna el descubrimiento de los $n a-$ notubos de carbono de pared múltiple (MWCNTs) a lijima por su famoso trabajo publicado en la revista Nature de título: "Helical microtubules of graphitic carbón" (10). Sin embargo, existe un elevado número de publicaciones previas que tratan sobre este tipo de sistemas, cuyos autores han quedado prácticamente en el olvido.

En el editorial de la revista Carbon, M. Monthioux y V. L. Kuznetsov actuando como editores invitados escribieron un artículo cuyo título: "Who should be given the credit for the discovery of carbón nanotubes?" es lo suficientemente explícito (11). En este editorial, se da el crédito debido a A. Oberlin quien, en 1976, mostró una imagen TEM de un nanotubo de carbono que, de acuerdo a su diámetro de unos $5 \mathrm{~nm}$ debía ser, probablemente, de pared múltiple (12).

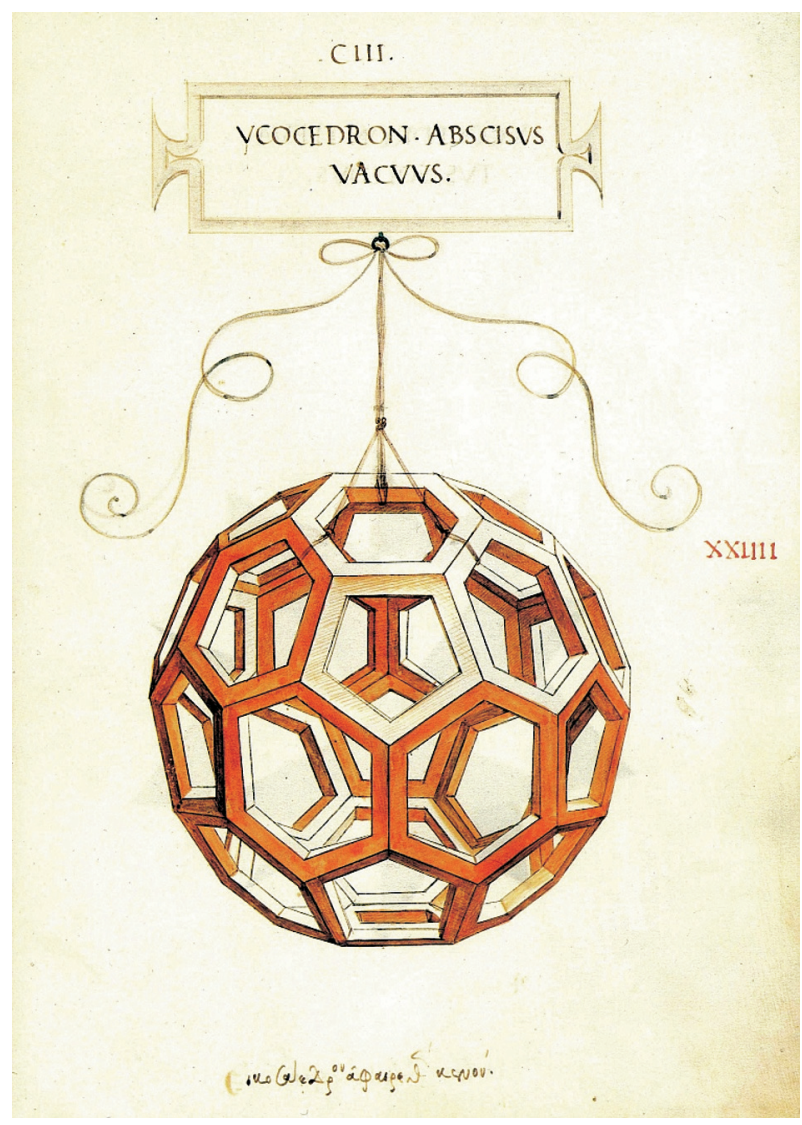

Figura 3. Ycocedron Abscisus Vacuus 
No obstante, la primera mención de la posibilidad de formar filamentos de carbono por descomposición térmica de hidrocarburos gaseosos (metano) fue descrita ya en 1889! (13) Aunque existen algunas otras publicaciones de esos años, no es posible hacer una asignación inequívoca debido a las limitaciones de los microscopios de la época. Ha sido con el desarrollo del primer microscopio de transmisión electrónica (TEM) comercial a partir de 1939, cuando se ha logrado establecer la morfología y textura interna de los filamentos de carbono. Fue un grupo de investigadores rusos quienes en 1952 aportaron las primeras evidencias mediante TEM de la formación de tubos de carbono (seguramente de pared múltiple conteniendo de 15 a 20 capas) (14). Naturalmente, la resolución de los TEM de la época no permitió distinguir el número de capas presentes en el nanotubo de carbono (Figura 4).

Pnc. 7 $\times 20.000$

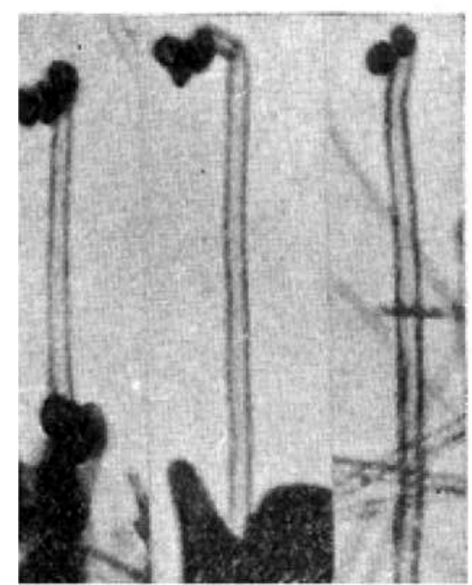

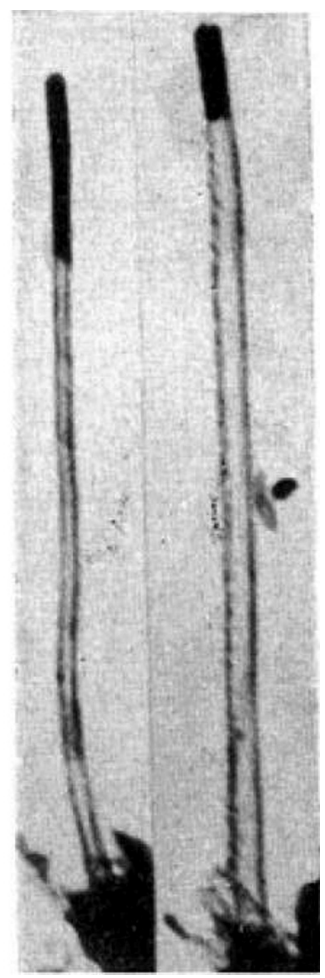

Figura 4. Los nanotubos de carbono fueron observados porRadushkevich y Lukyanovich usando TEM en 1952, pero no existía ningún proceso de fabricación conocido que permitiese la síntesis de cantidades macroscópicas de nanotubos de carbono (Ref. 14)
Curiosamente, la historia del descubrimiento de los nanotubos de carbono de pared sencilla no presenta polémica alguna ya que, queda bien establecido, que estos fueron descubiertos simultáneamente por los grupos de investigación de lijima e Ichihashi (15) (NEC Corporation) y Bethune et al. (16) (IBM) en 1993, con una diferencia en el envío de ambos manuscritos a la revista Nature de tan solo un mes. Curiosamente, la serendipia o descubrimiento fortuito tuvo que ver en ambos casos ya que la formación de los nanotubos de carbono de pared sencilla (SWCNTs) fueron resultado de la formación fallida de obtención de nanotubos de pared múltiple (MWCNTs) rellenos de metales de transición.

A la pregunta de por qué la comunidad científica parece haber descubierto los nanotubos de carbono en 1991 cuando ya eran conocidos cuarenta años antes, Monthioux y Kuznetsov dan dos razones de peso: la primera es que la meta principal de la comunidad científica implicada en estos sistemas de carbono era comprender su mecanismo de crecimiento e impedir su formación en la industria del carbón y del acero, así como en los canales de refrigeración de los reactores nucleares. Temas estos nada excitantes para los físicos! Además, esta comunidad científica estaba desvinculada de las revistas habituales de química. La segunda razón tiene que ver con la madurez de la ciencia, no solo en la evolución de las herramientas de medida como el TEM sino, aún más importante, con el hecho de que las mentes de los científicos implicados aún no pensaban en términos "nano".

Todo, pues, parece cristalizar en la publicación de lijima de 1991, en una revista del más alto prestigio científico y divulgación universal en un momento en que la "nanociencia" surge con gran fuerza.

\subsection{Grafenos}

El descubrimiento de los grafenos puede ser considerado más ortodoxo desde el punto de vista científico. Para comprender el grafeno es preciso conocer el grafito, el cual se conoce como mineral desde hace más de 500 años y que ya era utilizado en la edad media de forma parecida a como utilizamos hoy los lapiceros. Entre las múltiples propiedades del grafito destacan su elevada conductividad eléctrica y térmica a lo largo del plano, así como una elevada dureza mecánica. Las propiedades del grafito, al estar constituido 
por láminas de carbono con hibridación $\mathrm{sp}^{2}$ unidas débilmente entre sí, se caracterizan por su elevada anisotropía. Sus propiedades químicas se han centrado, especialmente, en reacciones de intercalación y reacciones redox al poder actuar como agente oxidante o reductor.

Aunque se han llevado a cabo diferentes estudios dirigidos a separar un número cada vez menor de capas de grafeno a partir de grafito, el impulso definitivo en el estudio de los grafenos se produjo en 2004 cuando Andre Geim y el que fuera su alumno de doctorado, Konstantin Novoselov, de la Universidad de Manchester, aislaron las primeras muestras de grafeno a partir de grafito mediante un proceso de exfoliación mecánica (17). El proceso es muy simple y consistió en la exfoliación de láminas de grafeno mediante el uso de una cinta de celofán, permitiendo, así, un acceso fácil a este material en el que se han depositado tantas expectativas (Figura 5).

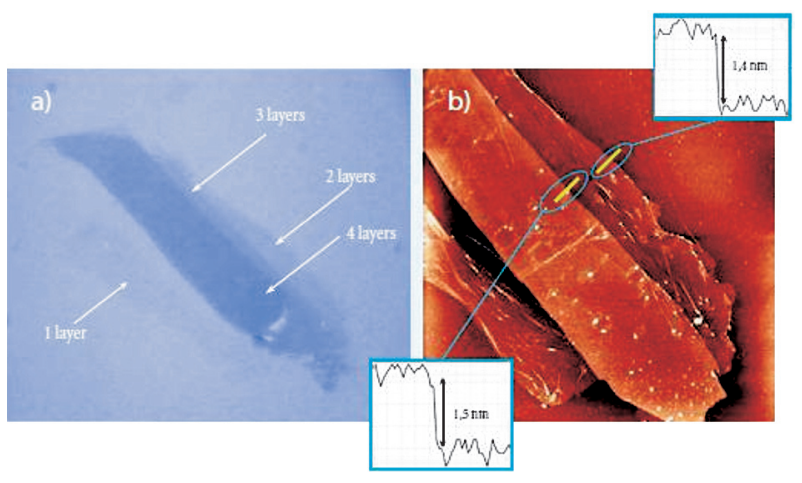

Figura 5. a) Imagen de luz blanca de una multicapa de grafeno. Tamaño de imagen $120 \times 110$ micras. b) Imagen AFM de la misma muestra con los perfiles correspondientes. Tamaño de barrido $50 \times 50$ micras

El interés en los grafenos ya ha superado el ámbito puramente científico y ha interesado a la sociedad en general por sus múltiples posibles usos y aplicaciones. Baste como ejemplo el artículo publicado a dos páginas por el diario El País el pasado 6 de agosto de 2010 escrito por el periodista Miguel Calzada y por la investigadora del CSIC Elsa Prada, titulado: "Proximamente en sus pantallas: el grafeno", aludiendo, de forma quizás algo exagerada, a las aplicaciones de este material de carbono en múltiples facetas (chips, ordenadores, baterías, sensores, electrodos, etc.) y anunciando la fabricación de la primera pantaIla táctil que puede enrollarse y doblarse de grafeno por investigadores coreanos. Simultáneamente, IBM anuncia la fabricación de chips de grafeno que son 10 veces más veloces que los de silicio.

Resultado de esta expectación e interés ha sido la reciente concesión del Premio Nobel de Física 2010 a sus descubridores (Andre Geim y Konstantin Novoselov) el pasado mes de octubre (18) (http://nobelprize.org/nobel_prizes/ physics/laureates/2010/). Sorprendentemente, han bastado seis años desde la descripción de este procedimiento experimental, conceptualmente muy simple, para que sus descubridores hayan recibido tan importante galardón. Sin embargo, no hay duda de la gran revolución que esta nueva forma de carbono ha provocado en la ciencia mundial, siendo actualmente el grafeno uno de los materiales más estudiados tanto en sus aspectos fundamentales como en sus posibles aplicaciones.

\section{Fullerenos: 25 años deSDE SU DESCUBRIMIENTO}

Los fullerenos, por su singular geometría esférica y propiedades electrónicas y mecánicas, han sido objeto de intensa investigación desde su descubrimiento en 1985. Desde entonces, se han publicado un elevado número de artículos de revisión y libros sobre su reactividad química, así como de las propiedades y aplicaciones que presentan en áreas como la ciencia de materiales o la biomedicina (19). Sin embargo, todavía es posible descubrir nuevas reacciones en la superficie del fullereno, las cuales son de interés para comprender la reactividad de los nuevos alótropos de carbono, cuyas propiedades pueden jugar un importante papel en el desarrollo de diferentes disciplinas. En el presente artículo se destacarán algunos ejemplos recientes que, según el autor, suponen una novedad y un avance real que puede ayudar en el conocimiento de las propiedades y comportamiento químico de las nuevas nanoestructuras de carbono.

\subsection{Fullerenos quirales a la carta}

Las excepcionales propiedades químicas, electroquímicas y fotofísicas de los fullerenos les hacen ser unos candidatos ideales para el desarrollo de la denominada electrónica mo- 
lecular orgánica. Su posible aplicación en la obtención de dispositivos fotovoltaicos o cables moleculares, hacen que su estudio sea muy atractivo. Sin embargo, también son de interés en química médica y biotecnología donde han demostrado su eficacia como inhibidores de varias enzimas, en procesos de neuroprotección y transfección, presentando actividad citotóxica sobre células tumorales e incluso provocando la ruptura del DNA con irradiación con luz visible.

Sin embargo, y a pesar de la importancia de la quiralidad en estos nuevos derivados de carbono, la síntesis y el empleo de fullerenos quirales en la búsqueda de nuevas propiedades biológicas ha estado muy limitada. Normalmente, se han utilizando materiales de partida ya quirales o se han obtenido algunos fullerenos quirales a través de largos y costosos procesos de separación cromatográfica extraordinariamente cara y limitada.

En nuestro grupo de investigación se ha desarrollado un método que permite, por primera vez, la funcionalización altamente selectiva de fullerenos en condiciones de reacción suaves mediante el empleo de metales, tales como plata o cobre, y de ligandos quirales en cantidades catalíticas. Esto, ha permitido tener un elevado control de la estereoquímica de los productos resultantes. En particular, de la estereoquímica de las denominadas fulleropirrolidinas, quizás el derivado más común y asequible de los fullerenos (Figura 6) (20).

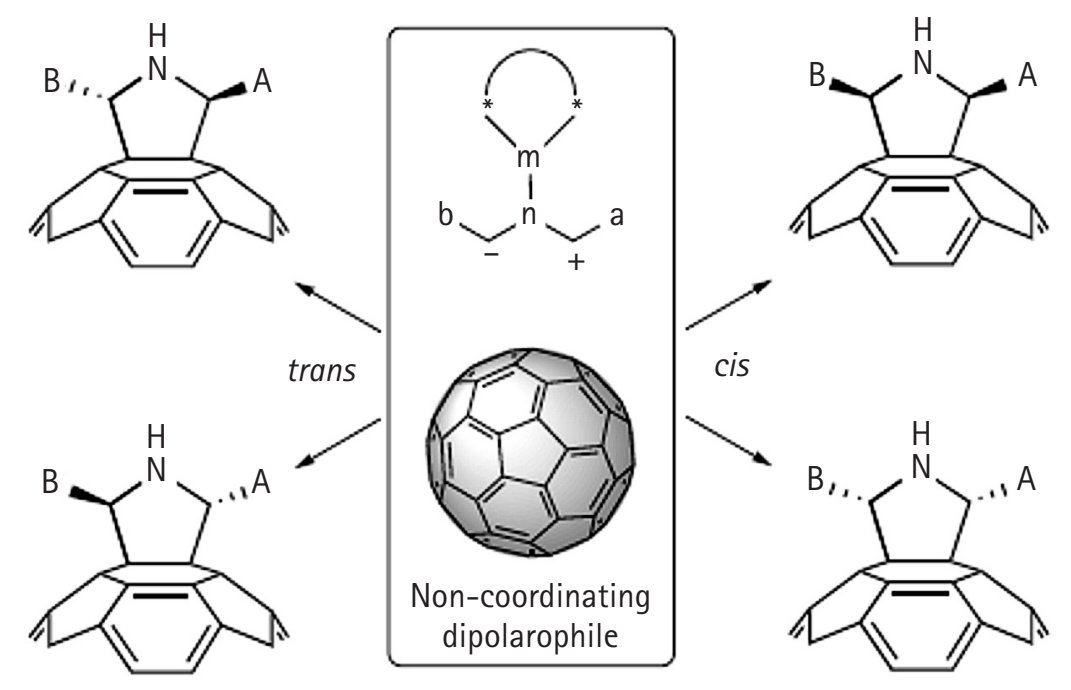

Figura 6. Iluros de azometino $N$-metalados se cicloadicionan al fullereno $C_{60}$ con un control en el resultado de la diastereo- $y$ enantioselectividad

En particular, la combinación adecuada de metal y ligando es capaz de modular en diferentes maneras el sentido de la inducción quiral y obtener fullerenos de configuración absoluta opuesta, con excesos enantioméricos alrededor del $90 \%$. Cabe destacar que tal resultado es aún más llamativo por ser la primera vez que se describe una reacción con catalizadores quirales empleando un reactivo no coordinate, como el fullereno $\mathrm{C}_{60}$. En este sentido, el empleo del ion acetato como contra-anión del metal catalítico ha tenido un papel fundamental puesto que, además de actuar como base, se coordina al complejo catalítico permitiendo así una mejor discriminación enantiofacial. En definitiva, que en cierto modo, es posible preparar fullerenos quirales a la carta mediante la elección cuidadosa del coctel catalítico adecuado.

Esta nueva metodología, constituye un importante avance en la química de los fullerenos y abre nuevas vías para la obtención de nanoestructuras de carbono quirales de interés en diferentes disciplinas científicas como la química médica o incluso la electrónica molecular orgánica. 


\subsection{Fullerenos para células fotovoltaicas}

Las aplicaciones fotovoltaicas representan, $\sin$ duda, la aplicación más realista de los fullerenos, en donde sus propiedades electrónicas y fotofísicas juegan un papel fundamental.

La energía es, sin duda, el problema más importante que afecta al humanidad. La que podemos denominar "época del fuego" en la que nuestra civilización se ha basado desde su origen está Ilegando a su fin. El ser humano ha quemado diferentes combustibles desde tiempos pasados $y_{1}$ en particular durante los dos últimos siglos, la combustión de combustibles fósiles basados en el carbono ha originado uno de los problemas más importantes a los que se enfrenta nuestra civilización. Como consecuencia de las cantidades ingentes de producción de $\mathrm{CO}_{2}$ resultante de la combustión, hechos tales como la elevada polución, calentamiento global y degradación del planeta hacen necesario el advenimiento de una nueva era basada en el uso de energías renovables no contaminantes que garanticen la subsistencia de nuestro planeta.

En este sentido, el Sol, considerado como un reactor gigante de fusión nuclear, representa la fuente más potente de energía disponible en todo nuestro sistema solar y, por tanto, su uso para suministrar energía a nuestro planeta representa, actualmente, uno de los retos más importantes de la ciencia actual. Como es bien sabido, la energía recibida del Sol es de unos 120.000 TW (5\% ultravioleta, 43\% visible y $52 \%$ infrarroja), lo que supera en más de mil veces la energía consumida en el planeta durante todo un año.

Los fullerenos y sus derivados han sido utilizado con éxito como aceptores de electrones en la preparación de célu- las solares de heterounión masiva (Bulk Heterojunction Solar Cells, BHJ). De hecho, la combinación del derivado de fullereno con MDM0-PPV \{poli[2-metoxi-5-(30,70dimetiloctiloxi)]-1,4-fenilenvinileno\} y P3HT [poli-(3hexiltiofeno)] como polímeros comerciales semiconductores, han dado lugar a la mayor parte de células solares de tipo BHJ estudiadas hasta el momento. De hecho, hasta hace poco tiempo, la combinación PCBM/P3HT (regiorregular) ha permitido acceder a células con conversión de energía del orden del 5\% (21).

En nuestro grupo de investigación hemos llevado a cabo la preparación de diferentes derivados de fullerenos para la construcción de dispositivos fotovoltaicos. De hecho, con derivados de difenilmetanofullereno (DPMs) hemos alcanzado conversiones de energía del orden del 2,3\% con MDM0-PPV (22) y del 3,0\% con P3HT (23).

Actualmente, se está desarrollando un gran esfuerzo en la preparación de nuevos polímeros de bajo gap (diferencia de energía HOMO-LUMO) que muestren una mejor absorción en la zona del visible y mejor morfología en su mezcla con el derivado de fullereno. Así, por ejemplo, se han preparado derivados de [3,4-b]thiofeno y benzoditiofeno (PTBs) con valores tan bajos como $1.6 \mathrm{eV}$. Una optimización posterior se ha llevado a cabo con el nuevo PTB en el que la presencia de los átomos de flúor conduce a un menor gap y una fuerte absorción en el visible desde 550 a $750 \mathrm{~nm}$. Interesantemente, la mezcla con el derivado de $C_{70}$. [70]PCBM, se ha conseguido construir células fotovoltaicas con una conversión de energía solar en energía eléctrica del 7,40\% (Figura 7). Este valor representa, hasta ahora, el valor más alto de conversión de energía conseguido en una célula solar de tipo polimérico (24).
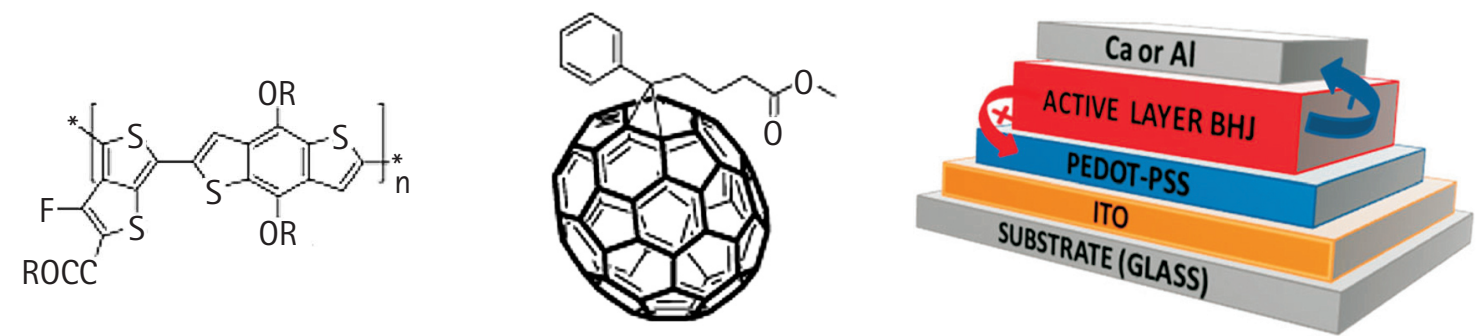

Figura 7. Polímero semiconductor PTB7 y derivado de fullereno [707PCBM. Su mezcla ha conducido a un material que presenta el valor más alto de eficiencia (7,4\%) conseguido en una célula fotovoltaica de tipo polimérico 
Aunque algunos de los derivados de fullereno preparados hasta ahora han mostrado una buena función en la formación de dispositivos fotovoltaicos, la síntesis de nuevos fullerenos con mejor absorción en el visible y valores del LUMO más altos es, actualmente, un reto para todos aquellos químicos implicados en el estudio de células fotovoltaicas basadas en los fullerenos.

\section{Nanotubos de carbono}

Aunque los nanotubos de carbono (CNTs), tanto de pared múltiple (MWCNTs) como de pared sencilla (SWCNTs) presentan propiedades electrónicas, mecánicas y químicas muy interesantes, en este apartado nos centraremos exclusivamente en los nanotubos de pared sencilla (SWCNTs) por ser, sin duda, los más estudiados hasta el momento.

Formalmente, los nanotubos de pared sencilla están formados por una lámina de grafeno curvada y cerrada, mientras que los MWCNTs contienen varias unidades de nanotubos de pared simple concéntricos. La estructura de los nanotubos de pared simple (SWCNTs) viene determinada por la forma en que se enrolla sobre sí misma la red hexagonal de grafeno, dando lugar a tres tipos diferentes de nanotubos, los de tipo sillón, de carácter metálico y aquiral, los de tipo quiral, de carácter semiconductor y los de tipo zigzag de carácter aquiral (25).

Los CNTs se encuentran habitualmente formando racimos o agregados de diferente diámetro, de tal forma que cada tubo puede tener diferente longitud y diámetro y presentan defectos tanto en los extremos como en la pared lateral. En general, los nanotubos no son solubles en disolventes orgánicos habituales, aunque se ha conseguido formar dispersiones de SWCNTs en medios muy polares como $\mathrm{N}, \mathrm{N}$-dimetilformamida (DMF), $\mathrm{N}$-metilpirrolidina y hexametilfosforamida (HMPA). La funcionalización química ofrece la posibilidad de incorporar fragmentos orgánicos a los nanotubos, que pueden contribuir a aumentar su solubilidad. La singular estructura de los CNTs nos ofrece una amplia gama de posibilidades para llevar a cabo esta funcionalización (26). Así los CNTs pueden ser modificados químicamente a través de diferentes aproximaciones como son: i) funcionalización mediante enlaces covalente sobre la pared lateral o los extremos, ii) funcionalización mediante interacciones no covalentes, y iii) inclusión endoédrica o interior.

\subsection{Crecimiento de CNTs en ausencia de catalizador metálico}

El interés de los CNTs se centra fundamentalmente en las excepcionales propiedades eléctricas, térmicas y mecánicas que presentan. De hecho, con un módulo de Young entorno a $1 \mathrm{TPa}$, son unas 100 veces más fuertes que el acero, presentan la conductividad térmica más alta conocida y sus propiedades electrónicas han encontrado aplicación en una variedad de aplicaciones tales como emisión de efecto campo, STM, nanodispositivos electrónicos, etc.

Actualmente, los CNTs de pared sencilla se preparan por tres diferentes métodos: i) ablación láser, ii) descarga en arco eléctrico y iii) deposición química de vapor (CVD). Mientras que los dos primeros métodos utilizan grafito como material de partida, el método de CVD utiliza moléculas pequeñas tales como metano, acetileno, alcoholes y monóxido de carbono. Sin embargo, común a todas estas técnicas es el uso de metales de transición como catalizadores (normalmente $\mathrm{Fe}, \mathrm{Ni}, \mathrm{Co}$ y sus aleaciones). La razón es la creencia de que para el crecimiento del CNT es indispensable la formación de carburos metálicos. Sin embargo, la presencia de estos catalizadores metálicos resulta prácticamente imposible de eliminar completamente después de la producción del CNT, siendo una interferencia extremadamente perjudicial para la práctica totalidad de las aplicaciones de los CNTs tanto en el ámbito de la ciencia de materiales como en las aplicaciones biológicas donde, las nanopartículas (NPs) metálicas formadas perturban, frecuentemente, los dispositivos fabricados o los análisis realizados.

En estudios paralelos recientes, Ren y Cheng et al. (27) y Huang et al. (28) han preparado CNTs en ausencia de metales! Este hecho, en el primer caso, está basado fundamentalmente en el uso de la técnica de CVD y una lámina de $\mathrm{SiO}_{2}$ depositada sobre $\mathrm{Si}$ o Si/SiO2 como sustrato, ha permitido la formación homogénea de SWCNTs utilizando un flujo de $\mathrm{CH}_{4}$ y $\mathrm{H}_{2}$ a $900^{\circ} \mathrm{C}$. Los autores sugieren la importancia de la formación de nanopartículas de $\mathrm{SiO}_{2}$ con un tamaño medio de $1,9 \mathrm{~nm}$ tras el tratamiento con $\mathrm{H}_{2}$ a $900^{\circ} \mathrm{C}$. En el segundo método, el argumento es básicamente el mismo, consistente en la formación, aunque de diferente modo, de nanopartículas de $\mathrm{SiO}_{2}$ como puntos de nucleación catalítica para el crecimiento de los SWCNTs. En ambos casos, la formación de NPs de $\mathrm{SiO}_{2}$ ha sido de- 
mostrada por AFM y resulta fundamental que tengan un tamaño menor de $2 \mathrm{~nm}$ para el crecimiento del nanotubo de carbono.

Una primera cuestión básica que surge es sobre el mecanismo de formación de los nanotubos en ausencia de metal, ya que es precisa la descomposición previa de los materiales de partida para el crecimiento del CNT, algo a priori imposible de conseguir con $\mathrm{SiO}_{2}$. Sin embargo, se ha sugerido que la alta movilidad de los átomos de $\mathrm{Si}$ y $\mathrm{O}$ en el $\mathrm{SiO}_{2}$ fundido a $900^{\circ} \mathrm{C}$ causando defectos con la capacidad catalítica para descomponer hidrocarburos.

En cualquier caso, la formación de CNTs libres de metal es de importancia fundamental para resolver quizás el problema más grave para el uso generalizado de CNTs, abriendo así el camino a su aplicación en múltiples campos.

\subsection{Transformación de CNTs en grafeno}

Como se discutirá en el apartado siguiente, el grafeno ha logrado captar la atención de la comunidad científica por sus potenciales propiedades en el ámbito de la nanoelectrónica. Aunque se siguen diferentes métodos de producción de grafenos, estos son, en general, poco controlables en lo que se refiere a tamaño y homogenei- dad de las muestras obtenidas. Pues bien, recientemente, se ha descrito de forma simultánea por dos grupos de investigación la obtención de tiras o cintas de tamaño nanométrico, "nanocintas", de grafeno mediante la apertura controlada de nanotubos de carbono de pared múltiple (MWCNTs) (29).

En la aproximación del grupo norteamericano de Tour, la suspensión de MWCNTs en ácido sulfúrico es sometida a tratamiento oxidante con $\mathrm{KMnO}_{4}$. Las nanocintas resultantes son muy solubles en agua y otros disolventes polares. Los autores proponen un mecanismo de apertura del nanotubo de carbono a modo de cremallera. El proceso de apertura comenzaría con la formación de un ester de manganato por adición a un doble enlace de la pared del nanotubo. La posterior oxidación estaría facilitada por el medio deshidratante que llevaría a la formación de un defecto de dicetona con dobles enlaces adyacentes, más reactivos, que dirigirian el siguiente ataque, favorecido por aspectos de tipo estérico. El proceso continuaria hasta la apertura total del nanotubo, generando así la nanocinta de grafeno.

Este proceso ha sido observado igualmente en los naotubos de pared sencilla (SWCNTs), si bien ha resultado ser algo más difícil de llevar a cabo (Figura 8).

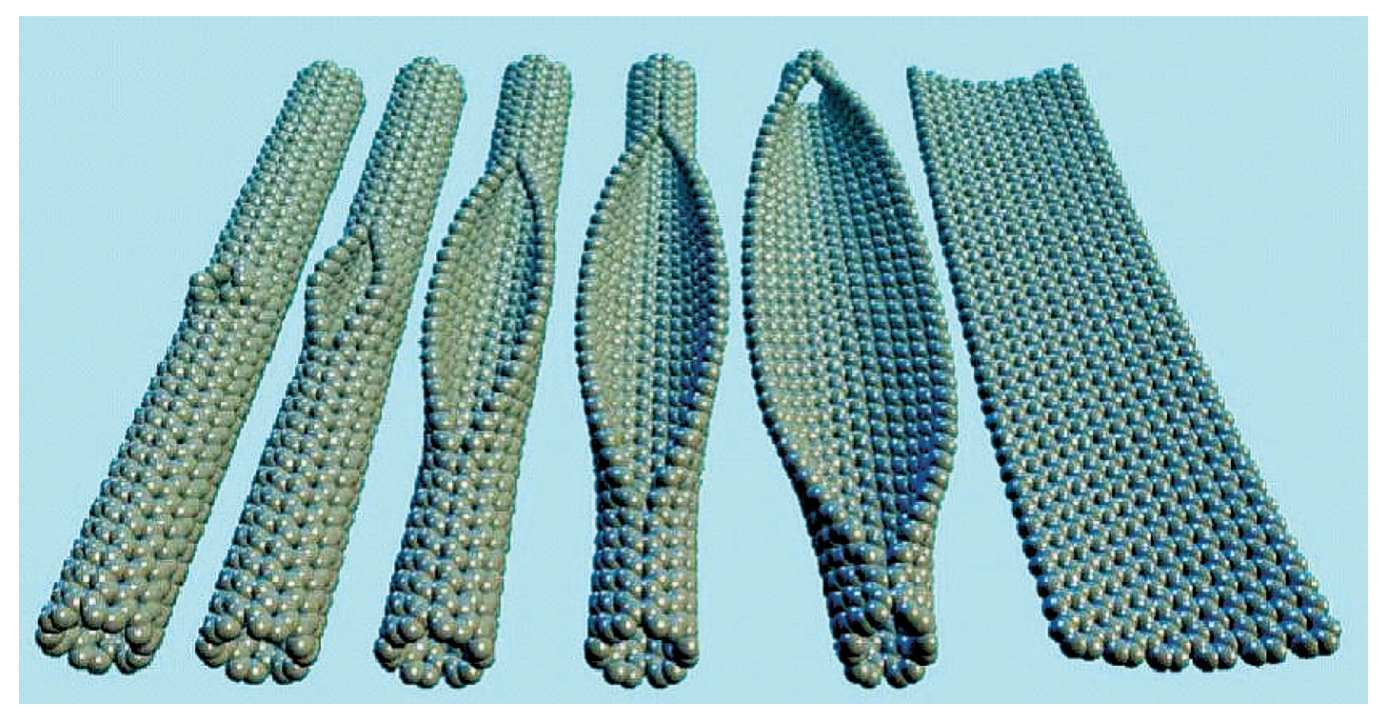

Figura 8. Representación de la apertura de un nanotubo de carbono a una cinta de grafeno por tratamiento oxidante (tomado de ref. 28 ) 
Aunque cabe pensar que el grafeno resultante queda funcionalizado con grupos que contienen oxígeno en sus bordes, este problema puede ser solventado, en parte, mediante un tratamiento con hidracina como agente reductor. Las nanocintas dde grafeno obtenidas son observads perfectamente por microscopia de fuerza atómica (AFM) y microscopia electrónica de barrido (SEM).

El método de Dai es, en esencia, parecido, si bien la apertura del nanotubo se lleva a cabo mediante la deposición del nanotubo en una capa polimérica de polimetacrilato de metilo, quedando embebido parcialmente. Esto permite que una parte del nanotubo quede expuesta a la posterior acción de un plasma de $\operatorname{Ar}$ a $10 \mathrm{~W}$ que actúa abriendo el nanotubo de carbono y formando nanocintas de grafeno muy regulares con anchura de la cinta del orden de 10$20 \mathrm{~nm}$.

Aunque los grafenos obtenidos por este procedimiento presentan algunas características algo inferiores a los obtenidos por exfoliación mecánica de grafito, es posible acceder, por vez primera a grafenos de tamaños controlados y regulares que, por su forma de cinta o de tira, pueden ser de gran interés en aplicaciones en nanoelectrónica tales como nanotransistores. Lamovilidad como transportadores de carga de estas nanocintas, aunque 10 veces menores que las medidas para grafenos bidimensionales, están entre las más altas medidas para un material hasta el momento, lo que puede llegar a ser una interesante alternativa al silicio.

\section{Grafenos}

Los grafenos se han convertido en los últimos años en el material estelar y objeto de deseo por parte de la comunidad científica debido, además de su potencial en futuras aplicaciones tecnológicas, al hecho mismo de ser el primer material bidimensional de anchura atómica, lo que supone ya un reto en términos de física fundamental (30). Debido a su inusual espectro electrónico, el grafeno ha conducido al surgimiento de un nuevo paradigma en la física de la materia condensada, donde fenómenos relativistas cuánticos, no observables en la física de altas energías, pueden ahora ser comprobados en el laboratorio.
Este nuevo material, independientemente de sus aplicaciones tecnológicas reales que se concreten en nuevos dispositivos para el avance tecnológico de nuestra sociedad, supone el poder acceder al estudio fundamental de la materia en un estado prácticamente desconocido hasta ahora (31). De hecho, hace más de 70 años, Landau y Peierls argumentaron que, estrictamente, los cristales bidimensionales (2D) eran termodinámicamente inestables y no podian existir. El argumento se basaba en el hecho de que una contribución divergente de fluctuaciones térmicas en estructuras cristalinas de baja dimensión deberían conducir a tales desplazamientos de átomos que serían comparables a las distancias interatómicas a cualquier temperatura finita (32). Por tanto, las monocapas atómicas han sido conocidas, hasta ahora, como parte integral de estructural tridimensionales (3D). Sin la estructura 3D, los materiales de estructura 2D no podían existir!

Esta situación ha cambiado drásticamente con la llegada de los grafenos (y otros materiales 2D tales como las monocapas de nitruro de boro). Pero los cristales 2D obtenidos, no solo son estables sino que, además, muestran una elevada calidad cristalina. Este hecho puede observarse perfectamente en el grafeno, donde los transportadores de carga pueden viajar miles de distancias interatómicas sin difundir.

Por tanto, el grafeno representa un nuevo "tipo de material" cuyo estudio permitirá avanzar en el conocimiento humano sobre la naturaleza que nos rodea. En los epígrafes siguientes se discutirán, a modo de ejemplos representativos algunos casos que, siendo de interés general, han llamado la atención de este autor. Naturalmente, este es un planteamiento subjetivo y el lector debe completar esta provocación que supone la presentación de estos materiales excepcionales con la lectura de las magníficas revisiones y literatura original existente sobre ellos.

Quisiera, finalmente, Ilamar la atención sobre un método de obtención de grafenos que se está desarrollando con gran vigor durante los últimos cuatro años. Se trata del crecimiento epitaxial de grafeno sobre diferentes sustratos metálicos cuya estructura geométrica y electrónica puede ser estudiada mediante STM (33). La interacción química entre la monocapa de grafeno y el sustrato metálico es muy 
variada, oscilando desde interacciones muy fuertes como el Ru (0001) (34) a muy débiles, como sobre $\operatorname{Ir(111)~(35).~}$

De hecho, la adsorción de moléculas orgánicas sobre superficies cristalinas bien definidas en condiciones de ultra-alto vacio es actualmente un campo de investigación de creciente importancia en el que participamos desde IMDEA-Nanociencia de forma muy activa (36). Las moléculas orgánicas depositadas sobre superficies metálicas a menudo modifican sus características geométricas y electrónicas debido a las interacciones con el sustrato y viceversa (37).

En este sentido, el crecimiento de una monocapa de grafeno sobre $\operatorname{Ir}(111)$ desacopla electrónicamente las moléculas orgánicas adsorbidas sobre el sustrato metálico, permitiendo, así, el estudio de la auto-organización de moléculas sobre superficies. Recientemente, y a modo de ejemplo, hemos llevado a cabo este estudio con moléculas fuertemente aceptoras de electrones derivadas de tetraciano-p-quinodimetano (TCNO), en donde las interacciones moleculares dictan el orden molecular sobre el grafeno (Figura 9) (38).

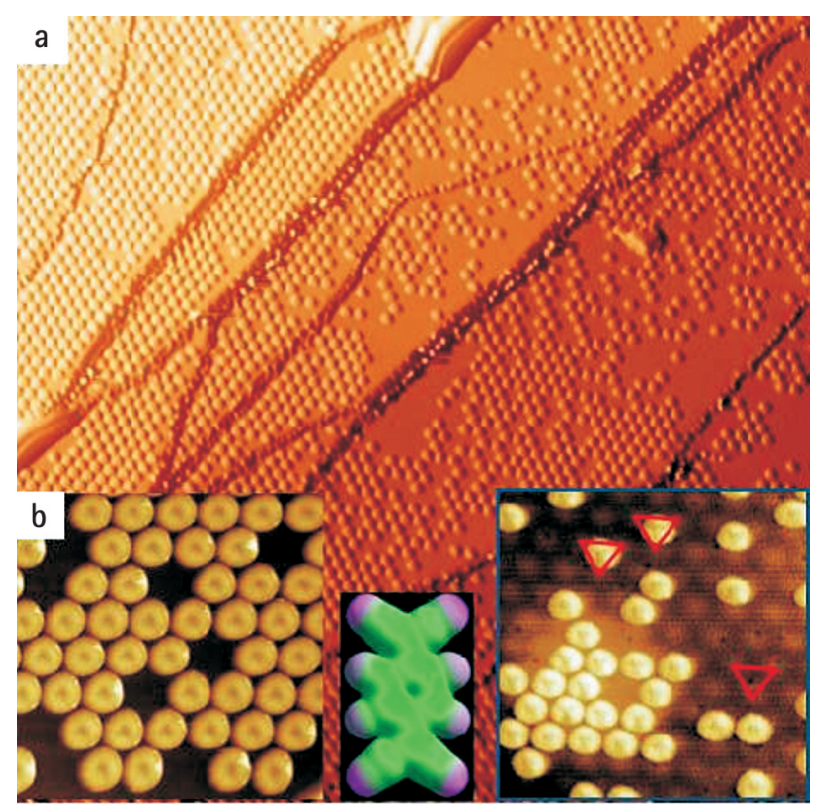

Figura 9. a) Imagen STM $(120 \mathrm{~nm} \times 80 \mathrm{~nm})$ tomada a $77 \mathrm{~K}$ después de depositar $F_{4}$-TCNQ sobre grafeno $/ \operatorname{Ir}(111)$; b) izquierda: $18 \mathrm{~nm} \times$ $18 \mathrm{~nm}(\mathrm{Vs}=-1.0 \mathrm{~V} ; \mathrm{It}=5 \mathrm{pA})$; derecha: $20 \mathrm{~nm} \times 20 \mathrm{~nm}(\mathrm{Vs}=-1.0 \mathrm{~V}$; $I t=5 \mathrm{pA}$ ). La molécula de $F_{4} T C N Q$ muestra su mapa electrostático (en púrpura para densidad de carga negativa y en verde para positiva)

\subsection{Transformación de grafenos a fullerenos}

Tal y como se ha comentado anteriormente, los fullerenos se obtienen a partir de grafito tras ser sometido a elevadas temperaturas. Sin embargo, su mecanismo de formación no está tan claramente establecido. Aunque se han llegado a establecer cuatro diferentes modos de formación de fullerenos a partir de grafito, todos ellos tienen en común el hecho de seguir una aproximación denominada bootmup (de abajo a arriba). Es decir, son los átomos de carbono generados a partir del grafito al ser sometido a altas temperaturas, o bien pequeños agregados atómicos, los que generan los fullerenos, cuya evolución se ha seguido cuando ha sido posible mediante medidas de espectrometría de masas de especies presentes en la fase gas (39).

Recientemente, Chuvilin, Khlobystov et al., han descrito en la revista Nature Chemistry la formación de una molécula de fullereno a partir de una pequeña lámina o copo (flake) de grafeno cuya evolución se ha podido seguir por vez primera mediante microscopia electrónica de transmisión (TEM) utilizando un haz de electrones de $80 \mathrm{keV}$ (40).

La alta energía del haz de electrones cuando interacciona con el grafeno puede provocar la fragmentación de la lámina en pequeños copos. Estos copos adsorbidos sobre el grafeno sufren una transformación posterior, que puede seguirse por TEM, que finaliza en la formación del fullereno (Fig. 10a,b).

La secuencia mostrada en la Figura 10 (c-h) muestra la evolución de la forma desde el copo de grafeno hasta la formación de la molécula de fullereno. Aparentemente, la pérdida de átomos de carbono en los bordes del grafeno es la etapa inicial en esta transformación al ser más lábiles. Esta pérdida conlleva una reconstrucción estructural a una configuración zig-zag más estable en los bordes del grafeno. Sin embargo, en los casos de copos de grafenos más pequeños, la reconstrucción conduce a la formación del fullereno de modo directo. Es evidente que el haz de electrones de alta energía produce la inestabilización del sistema que evluciona formando anillos pentagonales en los bordes, que favorecen la curvatura $y$, por tanto, la posterior formación de enlaces carbono-carbono.

Naturalmente, aún quedan aspectos que aclarar en este procedimiento, pero los cálculos y estudios realizados has- 

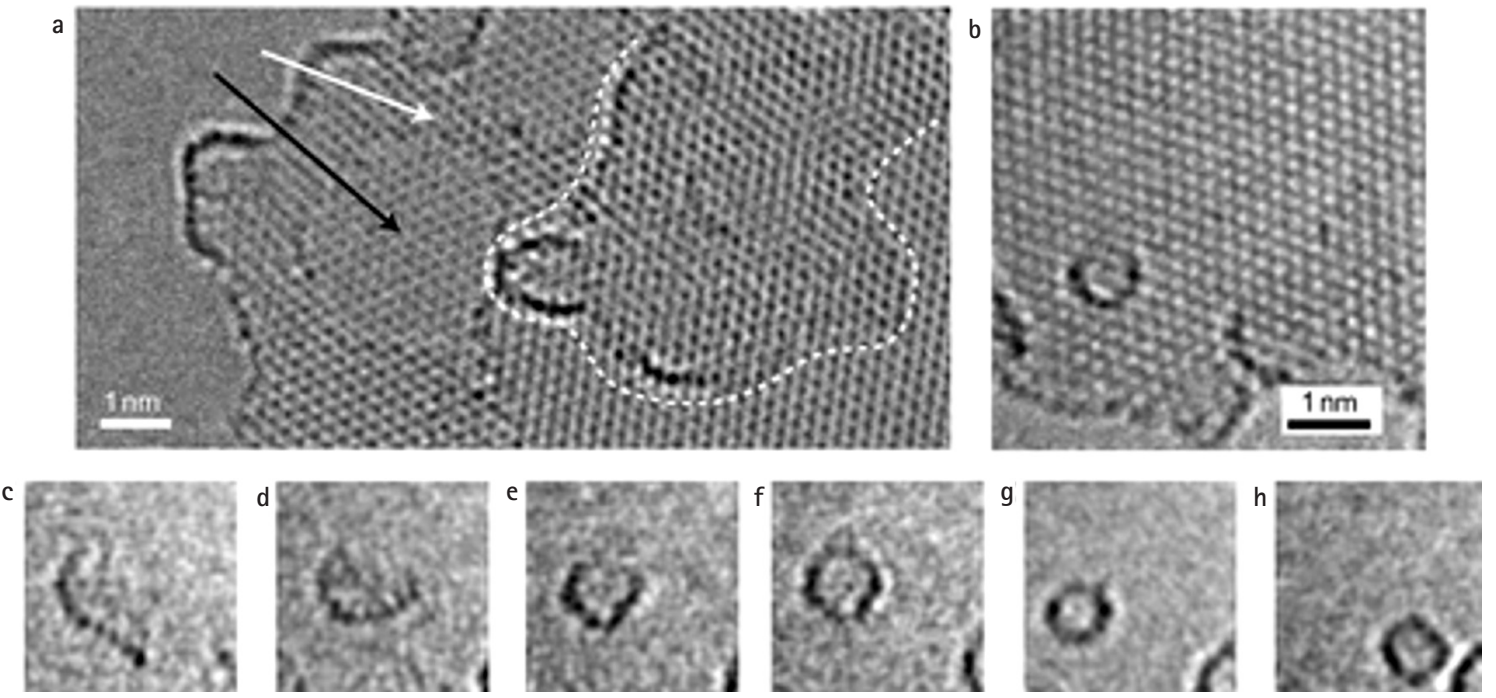

Figura 10. Imágenes TEM mostrando la formación de una molécula de fullereno a partir de una lámina de grafeno

ta ahora relacionan el tamaño del copo de grafeno con el tamaño del fullereno obtenido, por lo que grafenos por encima de 100 átomos de carbono deben ser mucho más difícil de formar desde el punto de vista energético.

Este método de formación de fullerenos constrasta con los conocidos hasta ahora, dado que no se precisa la disociación previa en átomos de carbono o pequeños agregados para la formación de la molécula de fullereno, tal y como muestra la secuencia de imágenes obtenidas mediante la técnica TEM.

Este interesante estudio no solo ha permitido comprender mejor la formación de fullerenos sino que, también, puede ser una alternativa realista a la producción de fullerenos.

\subsection{Química de grafenos. Evolución estructural durante la reducción química de óxido de grafeno}

Quiero concluir este artículo con un epígrafe dedicado a los aspectos más químicos del grafeno. En este sentido, aunque su "descubrimiento" ha sido el más reciente, ya existe un elevado número de artículos científicos en los que se ha estudiado el comportamiento y reactividad química de los grafenos. Naturalmente, para comprender algunos de estos estudios es preciso recordar al lector la química previamente desarrollada sobre el grafito como tal material y las múltiples reacciones de inclusión realizadas sobre el mismo.

Existen ya diferentes revisiones bibliográficas dedicadas a los grafenos y, también, a su química, a las cuales se recomienda que acuda el lector interesado en ampliar este tema. En este epígrafe pretendo, únicamente, mostrar un caso singular de reactividad química de grafenos. En concreto, el estudio teórico, apoyado por resultados experimentales publicado recientemente en la revista Nature Chemistry por Shenoy et al. (41), en donde se da respuesta al hecho de la presencia de oxígeno en las láminas de óxido de grafeno, una vez sometido a un proceso de reducción enérgica. Este hecho es de gran importancia dado que las propiedades del grafeno reducido se pierden, en gran parte debido a los defectos provocados por la presencia del oxígeno.

Entre los diferentes métodos de funcionalización química de grafenos, uno de los más estudiados se basa en el empleo de óxido de grafeno (G0), ya que es un material procesable en disolución y que puede ser depositado fácilmente en una amplia variedad de sustratos. Como es de suponer, el GO posee carbonos $s p^{3}$ unidos al oxígeno resultando 
un material eléctricamente aislante. La eliminación del oxígeno debe conducir a un material semiconductor. Sin embargo, todos los estudios realizados hasta el momento revelan que, independientemente del proceso seguido, el GO una vez sometido a un proceso de reducción ( $\mathrm{rGO}$ ) conduce a un material que contiene aproximadamente un $8 \%$ de oxígeno, lo que supone que en torno al $20 \%$ de los átomos de carbono presentan hibridación $s p^{3}$.

El oxígeno presente en el G0 suele encontrase principalmente como grupos epoxi e hidroxilo. Sin embargo, también puede darse la presencia de cetonas, fenoles $y_{1}$ en los bordes grupos carboxilo, anhídridos, lactonas, fenoles, pironas, etc.

El estudio realizado por Shenoy permite entender la razón de este hecho experimental. Mediante simulaciones de dinámica molecular ha mostrado que la reducción por tratamiento térmico conduce a la formación última de grupos carbonilo y éter, los cuales son termodinámicamente estables y no pueden ser eliminados posteriormente sin destruir la capa de grafeno. Esta importante conclusión ha sido confirmada por estudios de espectroscopía electrónica de rayos-X (XPS) realizada durante el proceso de templado térmico (Figura 11).
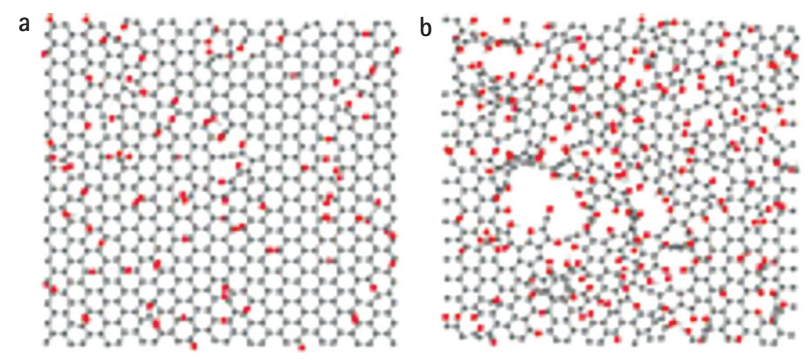

Figura 11. Morfología de láminas de óxido de grafeno reducido (rGO) con una concentración de $20 \%$ (a) y $33 \%$ (b) en forma de grupos hidroxilo y epoxi en una proporción de 3/2 después del templado térmico a $1500 \mathrm{~K}$

Aparentemente, los grupos hidroxilo se desorben de la superficie a temperaturas relativamente bajas, sin que se altere la estructura del grafeno. Por el contrario, los grupos epoxi, más estables, se desorben de la superficie de grafeno alterando su estructura significativamente. Los cálculos indican que la eliminación de átomos de carbono del grafeno se produce, principalmente, cuando ambos grupos hidroxilo y epoxi se encuentran próximos. El camino seguido por la reacción al someterla a un templado térmico concluye en la formación de grupos funcionales carbonilo y éter, que son termodinámicamente más estables. Estos cálculos teóricos, apoyados en medidas experimentales de XPS han permitido entender uno de los enigmas más curiosos que rodeaban la reactividad química de estos derivados de grafeno, lo que en principio limita por el momento, el posible uso de los óxidos de grafeno como materiales de partida para la preparación química de grafenos.

\section{Conclusiones}

En este artículo se ha pretendido dar a conocer más, si cabe, el misterioso mundo de los fullerenos y sus estructuras relacionadas, principalmente nanotubos de carbono y los más recientes grafenos. Comenzando por sus aspectos históricos, no siempre bien comprendidos, y siguiendo con algunos ejemplos recientes de la literatura que este autor de forma subjetiva considera relevantes por diferentes circunstancias.

Resulta sorprendente comprobar cómo las diferentes manifestaciones de los fullerenos, como alótropos de carbono, pueden transformarse entre sí, dando idea de la gran versatilidad de este átomo singular de la Tabla Periódica. Se ha visto como los nanotubos de carbono pueden transformarse en cintas de grafeno $y$, a su vez, como los grafenos pueden transformarse en fullerenos, en un baile de átomos de carbono que el científico empieza a comprender y a controlar. Sin duda que los fullerenos en general, a pesar de su relativamente reciente descubrimiento, llevan muchos años en nuestro planeta $y$, también, en el espacio interestelar formando parte de otros asteroides (42). Sin embargo, su observación solo ha sido posible, una vez más en ciencia, cuando las técnicas de medida y observación disponibles lo han permitido. En este sentido, las técnicas de microscopia electrónica (SEM, TEM) y de fuerza atómica (AFM) así como la microscopia de efecto túnel (STM) han participado de forma decisiva en estos descubrimientos y ya son, de hecho, una herramienta imprescindible en la investigación química.

Sin duda que el carbono, el elemento químico más próximo al ser humano, seguirá siendo a lo largo de este siglo actor principal en el desarrollo de las nuevas nanotecnologías a través de los alótropos ya conocidos y las nuevas y sorprendentes "nanoformas" que, seguramente, nos depara el futuro (43). 


\section{AGRADECIMIENTOS}

El autor expresa su más sincero agradecimiento a todos aquellos científicos cuyos trabajos se ven reflejados en este artículo $y_{1}$ especialmente, a aquellos de su grupo de investigación que con gran entusiasmo y dedicación han obtenido de forma brillante algunos de los resultados aqui expuestos. Quiero manifestar igualmente, mi agradecimiento a las instituciones que han financiado los proyectos de investigación relacionados con este trabajo. Al Ministerio de Ciencia e Innovación de España (CT2008-00795/BQU), Proyecto Consolider Ingenio 2010C-07-25200 en Nanociencia Molecular, La Comunidad de Madrid (Madrisolar-2, S2009/PPQ-1533) y la European Science Foundation (SOHYD, MAT2006-28170-E).

\section{REFERENCIAS}

(1) a) F. A. Carey "Química Orgánica" (6. Edición), McGraw Hill, 2006. Esta edición presenta en su portada la formación de una estructura de alótropo de carbono. b) Para una discusión más elegante sobre la capacidad del carbono para la creación de vida, véase: P. Levi, "The Periodic Table", Schocken Books, Random House Inc., New York, 1995, capítulo: Carbon, p. 224.

(2) H. W. Kroto, J. R. Heath, S. C. O'Brien, R. F. Curl, R. E. Smalley, Nature, 1985, 318, 162.

(3) a) Descubrimiento de nanotubos de carbono de pared múltiple: S. lijima, Nature 1991, 354, 56. Descubrimiento de nanotubos de carbono de pared sencilla: b) S. Iijima, T. Ichihashi, $\mathrm{Na-}$ ture 1993, 363, 603. c) D. S. Bethune, C. H. Klang, M. S. de Vries, G. Gorman, R. Savoy, J. Vasquez, R. Beyers, Nature 1993, 363, 605.
(4) K. S. Novoselov, A. K. Geim, S. V. Morozov, D. Jiang, Y. Zhang, S. V. Dubonos, I. V. Grigorieva, A. A. Firsov, Science, 2004, 306, 666.

(5) a) Para una revision reciente, véase: J. L. Delgado, M. A. Herranz, N. Martin, "The Nanoforms of Carbon", J. Mater. Chem. 2008, 18, 1417; b) "Nanoestructuras de carbono: un nuevo desafío científico", Anales de la RSEQ de Química, 2007, 103, 5.

(6) Las conferencias pronunciadas con motivo de la concesión del Premio Nobel han sido publicadas: a) H. Kroto, "Symmetry, Space, Stars, and C 60 ", Angew. Chem. Int. Ed., 1997, 36, 1578; b) R. E. Smalley, "Discovering the Fullerrenes", Angew. Chem. Int. Ed., 1997, 36, 1594 ; c) R. F. Curl, "Dawn of the Fullerenes: Conjeture and Experiment", Angew. Chem. Int. Ed., 1997, 36, 1566.

(7) W. Krätschmer, L. D. Lamb, K. Fostiropoulos and D. H. Huffman, Nature, 1990, 347, 354.

(8) E. Osawa, Kagaku (Kyoto), 1970, 25, 854 (en japonés). Chem. Abstr. 1971, 74, 75698v.

(9) P. A. Thrower, editor in chief, "Novel carbon materials - What if?", editorial de Carbon, 1999, 37, 1677.

(10) S. lijima, "Helical microtubules of graphitic carbon". Nature 1991, 354, 56.

(11) M. Monthioux, V. L. Kuznetsov, "Who should be given the credit for the discovery of carbon nanotubes?" Guest editorial, Carbon, 2006, 44, 1621.

(12) A. Oberlin, M. Endo, T. Koyama, "Filamentous growth of carbon through benzene decomposition". J. Cryst. Growth, 1976, 32, 335.

(13) T. V. Hughes, C. R. Chambers, US Patent 405480, 1889.

(14) L. V. Radushkevich, V. M. Lukyanovich, Zurn. Fisic. Chim. 1952, 26, 88.

(15) S. lijima, T. Ichihashi, "Single-shell carbon nanotubes of 1-nm diameter". Nature 1993, 363, 603. 
(16) D. S. Bethune, C. H. Kiang, M. S. De Vries, G. Gorman, R. Savoy, J. Vázquez, R. Beyers, Nature, 1993, 363, 605.

(17) A. K. Geim, K. S. Novoselov, "The rise of graphene", Nature Materials, 2007, 6, 183.

(18) Véase: R. Miranda, El País "Un experimento del viernes" 13 de octubre de 2010.

(19) Para algunos libros dedicados a fullerenos, véase: (a) D. M. Guldi, N. Martín Eds., Fullerenes: From Synthesis to Optoelectronic Properties, Kluver Academic Publishers, Dordrecht, Holanda, 2002. (b) A. Hirsch, M. Bettreich, Fullerenes, Chemistry and Reaction, WileyVCH, Weinheim, Alemania, 2005. (c) F. Langa, J.-F. Nierengarten Eds., Fullerenes. Principles and Applications, RSC, Cambridge, Reino Unido, 2007.N. Martín, "New Challenges in Fullerrene Chemistry", Chem. Commun. 2006, 2093-2104.

(20) S. Filippone, E. E. Maroto, A. Martín-Domenech, M. Suarez, N. Martin, "An efficient approach to chiral fullerene derivatives by catalytic enantioselective 1,3-dipolar cycloadditions", Nature Chem. 2009, 1, 578.

(21) Para una revisión reciente sobre materiales para células fotovoltaicas de naturaleza orgánica, véase: J. L. Delgado, P.-A. Bouit, S. Filippone, M. A. Herranz, N. Martín, Chem. Commun., 2010, 46, 4853. Otras revisions más generales: a) G. Dennler, M. C. Scharber, Ch. J. Brabec, Adv. Mater., 2009, 21, 1323; b) B. Kippelen, J.-J. Brédas, Energy Environ.Sci., 2009, 2, 251; c) B. C. Thompson, J. M. J. Fréchet, Angew. Chem., Int. Ed., 2008, 47, 58; d) S. Gunes, H. Neugebauer, N. S. Sariciftci, Chem. Rev., 2007, 107, 1324.
(22) I. Riedel, E. von Hauff, J. Parisi, N. Martín, F. Giacalone, V. Diakonov, Adv. Funct. Mater., 2005, 15, 1979.

(23) a) A. Sánchez-Diaz, M. Izquierdo, S. Filippone, N. Martín, E. Palomares, Adv. Funct. Mater., 2010, DOI: 10.1002/ adfm.201000549. b) H. J. Bolink, E. Coronado, A. Forment-Aliaga, M. Lenes, A. La Rosa, S. Filippone, N. Martín, J. Mater. Chem., 2010, DOI: 10.1039/b000000x. c) G. Garcia-Belmonte, P. P. Boix, J. Bisquert, M. Lenes, H. J. Bolink, A. La Rosa, S. Filippone, N. Martín, J. Phys. Chem. Lett., 2010, 1, 2566. d) M. Hallermann, E. Da Como, J. Feldmann, M. Izquierdo, S. Filippone, N. Martín, S. Jüchter, E. von Hauff, Appl. Phys. Lett., 2010, 97, 0233301.

(24) Y. Liang, Z. Xu, J. Xia, S.-T. Tsai, Y. Wu, G. Li, C. Ray, L. Yu, Adv. Mater., 2010, 22, E135.

(25) Para algunos libros dedicados a nanotubos de carbono, véase: a) V. N. Popov, P. Lambin, P. eds. "Carbon Nanotubes", Springer, Dordrecht, 2006. b) S. Reich, C. Thomsen, J. Maultzsch, eds. "Carbon Nanotubes: Basic Concepts and Physical Properties"; VCH: Weinheim, Germany, 2004. c) P. J. F. Harris, eds., "Carbon Nanotubes and Related Structures: New Materials for the Twenty-First Century"; Cambridge University Press: Cambridge, 2001. d) T. Akasaka, F. Wudl, S. Nagase, eds. "Chemsitry of nanocarbons", John Wiley \&t Sons, 2010. e) D. M. Guldi, N. Martín, eds. "Carbon Nanotubes and Related Structures"; VCH-Wiley, Weinheim, Germany, 2010.

(26) a) A. Hirsch, "Functionalization of single-walled carbon nanotubes", Angew. Chem. Int. Ed., 2002, 41, 1853; b) J. L. Barh and J. M. Tour, "Covalent chemistry of singlewall carbon nanotubes", J. Mater.
Chem., 2002, 12, 1952; c) S. Nigoyi, M. A. Hamon, H. Hu, B. Zhao, P. Bhomwik, R. Sen, M. E. Itkis and R. C. Haddon, "Chemistry of singlewalled carbon nanotubes", Acc. Chem. Res., 2002, 35, 1105; d) Y.P. Sun, K. Fu, Y. Lin and W. Huang, "Functionalized carbon nanotubes: Properties and applications", Acc. Chem. Res., 2002, 35, 1096; e) S. Banerjee, M. G. C. Kahn and S. S. Wong, "Rational chemical strategies for carbon nanotube functionalization", Chem. Eur. J., 2003, 9, 1898; f) D. Tasis, N. Tagmatarchis, V. Georgakilas and M. Prato, "Soluble carbon nanotubes", Chem. Eur. J., 2003, 9, 4000; g) C. A. Dyke and J. M. Tour, "Overcoming the insolubility of carbon nanotubes through high degrees of sidewall functionalization", Chem. Eur. J., 2004, 10, 812; h) S. Banerjee, T. Hemraj-Benny and S. S. Wong, "Covalent surface chemistry of single-walled carbon nanotubes", Adv. Mater., 2005, 17, 17; i) D. M. Guldi, G. M. A. Rahman, F. Zerbetto and M. Prato, "Carbon nanotubes in electron donor-acceptor nanocomposites", Acc. Chem. Res., 2005, 38, 871 ; j) D. Tasis, N. Tagmatarchis and A. Bianco, "Chemistry of carbon nanotubes", Chem. Rev., 2006, 106, 1105 ; k) D. M. Guldi, G. M. A. Rahman, V. Sgobba and C. Ehli, "Multifunctional molecular carbon materials - from fullerenes to carbon nanotubes", Chem. Soc. Rev., 2006, 35,471 ; I) D. M. Guldi, "Nanometer scale carbon structures for chargetransfer systems and photovoltaic applications", Phys. Chem. Chem. Phys., 2007, 9, 1400.

(27) B. Liu, W. Ren, L. Gao, S. Li, S. Pei, C. Liu, S. Jiang, H.-M. Cheng, J. Am. Chem. Soc., 2009, 131, 2082. 
(28) S. Huang, 0. Cai, J. Chen, Y. Qian, L. Zhang, J. Am. Chem. Soc., 2009, 131, 2094.

(29) a) D. V. Kosynkin, A. L. Higginbotham, A. Sinitskii, J. R. Lomeda, A. Dimiev, B. K. Price, J. M. Tour, Nature, 2009, 458, 872; b) L. Jiao, L. Zhang, X. Wang, G. Diankov, H. Dai, Nature, 2009, 458, 877.

(30) Para una revisión reciente, véase: M. J. Allen, V. C. Tung, R. B. Kaner, Chem. Rev., 2010, 110, 132.

(31) Otra revisión de grafenos: C. N. R. Rao, A. K. Sood, K. S. Subrahmnyam, A. Govindaraj, "Graphene: The new two-dimensional Nanomaterial", Angew. Chem. Int. Ed., 2009, 48, 7752.

(32) L. D. Landau, E. M. Lifshitz, "Statistical Physics", Part I, Pergamon, Oxford, 1980.

(33) a) T.A. Land, T. Michely, R.J. Behm, J.C. Hemminger and G. Comsa, Surf. Sci., 1992, 264, 261; b) A.T. N'Diaye, S. Bleikamp, P.J. Feibelman and T. Michely, Phys. Rev Lett., 2006, 97, 215501; c) Yu S. Devkov, M. Fonin and C. Laubschat, Appl. Phys. Lett. 2008, 92, 052506; d) S. Marchini, S. Günther and J. Wintterlin, Phys. Rev. B, 2007, 76, 075429.

(34) a) A. L. Vázquez de Parga, F. CaIleja, B. Borca, M. C. G. Passeggi, J. J. Hinarejos, F. Guinea and R. Miranda, Phys. Rev. Lett. 2008, 100, 90 056807; b) B. Borca, S. Barja,
M. Garnica, D. Sánchez-Portal, V. M. Silkin, E. V. Chulkov, C. F. Hermanns, J. J. Hinarejos, A. L. Vázquez de Parga, A. 95 Arnau, P. M. Echenique and R. Miranda, Phys. Rev. Lett., 2010, 105, 036804.

(35) I. Pletikosic, M. Kralj, P. Pervan, R. Brako, J. Coraux, A. T. N'Diaye, C. Busse and T. Michely, Phys. Rev. Lett., 2009, 102, 056808.

(36) L. Sánchez, R. Otero, J. M. Gallego, R. Miranda, N. Martín, "Ordering FuIlerenes at the Nonometer Scale on Solid Surfaces", Chem. Rev. 2009, 109, 2081.

(37) T.-Ch. Tseng, C. Urban, Y. Wang, R. Otero, S. L. Tait, M. Alcami, D. Écija, M. Trelka, J. M. Gallego, N. Lin, M. Konuma, U. Starke, A. Nefedov, A. Langner, C. Wolf, M. A. Herranz, F. Martín, N. Martín, K. Kern and R. Miranda, Nature Chem., 2010, 2, 374 .

(38) S. Barja, M. Garnica, J. J. Hinarejos, A. L. Vázquez de Parga, N. Martín, R. Miranda, "Self-organization of electron acceptor molecules on graphene", Chem. Commun., enviado para publicación.

(39) a) C. S. Yannoni, P. P. Bernier, D. S. Bethune, G. Meijer, J. R. Salem, "NMR determination of the bond lengths in $\mathrm{C}_{60}$ ". J. Am. Chem. Soc. 1991, 113, 3190; J. M. Hawkins, A. Meyer, S. Loren, R. Nunlist, "Statistical incorporation of carbon-13
${ }^{13} \mathrm{C}_{2}$ units into $\mathrm{C}_{60}$ (buckminsterfullerene)". J. Am. Chem. Soc. 1991, 113, 9394; T. W. Ebbesen, J. Tabuchi, K. Tanigaki, "The mechanistics of fuIlerene formation". Chem. Phys. Lett. 1992, 191, 336.

(40) A. Chuvilin, U. Kaiser, E. Bichoutskaia, N. A. Besley, A. N. Khlobystov, Nature Chem., 2010, 2, 450.

(41) A. Bagri, C. Mattevi, M. Acik, Y. J. Chabal, M. Chhowalla, V. B. Shenoy, Nature Chem., 2010, 2, 581.

(42) Muy recientemente, científicos de la Universidad de Western Ontario han descubierto en la nebulosa planetaria Tc 1, mediante el espectro infrarrojo del telescopio espacial Spitzer, que su espectro IR está dominado por las bandas de fullereno $C_{60} y_{1}$ en menor extensión, por $C_{70}$, ambos en forma neutra. Se estima que la cantidad de fullerenos presente en Tc 1 es alrededor del 1,5\% del carbono presente, y es equivalente a la masa de tres Lunas. Véase: E. J. Cami, J. Bernard-Salas, E. Peeters, S. E. Malek, "Detection of $\mathrm{C}_{60}$ and $\mathrm{C}_{70}$ in a young planetary nebula". Science doi: 10.1126/science.1192035 (2010).

(43) a) N. Martín, "El carbono y la nanotecnología del siglo XXI", El País, septiembre 2003. b) N. Martín, "Ladrillos moleculares", El País, octubre 2010. 